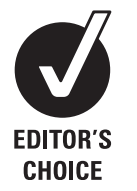

CHOICE
${ }^{1}$ Assistance Publique-Hôpitaux de Paris, Hôpital Européen Georges Pompidou, Department of Cardiology, University Paris Descartes, INSERM U 633, Paris, France

${ }^{2}$ Laboratoire de Chimie Analytique, E.A. 4041, Faculté de Pharmacie,

Châtenay-Malabry, France ${ }^{3}$ Centre de référence de la maladie de Fabry et des maladies héréditaires du tissu conjonctif, CHU Raymond Poincaré, Garches, France ${ }^{4}$ Department of Cardiology, Centre d'Investigation Clinique and Plateforme de Ressources Biologiques, Hôpital Henri Mondor, Créteil, France

${ }^{5}$ Department of Cardiology, Hôpital du Haut Lévèque,

Pessac, France

${ }^{6}$ Hôpital Cardiologique, Lille, France

${ }^{7}$ Centre de Recherche Clinique, Hôpital Dupuytren, Limoges,

France

${ }^{8}$ Laboratoire de Biochimie, $\mathrm{CHU}$ Raymond Poincaré, Garches, France

${ }^{9}$ Centre de référence des maladies cardiaques héréditaires, Hôpital de la Pitié-Salpêtrière, Paris, France ${ }^{10}$ Laboratoire de Génétique Médicale, CHU Raymond Poincaré (AP-HP), Garches, France

${ }^{11}$ University of Versailles - St Quentin en Yvelines, Versailles, France

\section{Correspondence to} Dr Albert A Hagège, Department of Cardiology, Hôpital Européen Georges Pompidou, 20 Rue Leblanc, 75015 Paris, France albert.hagege@egp.aphp.fr

Accepted 28 September 2010 Published Online First

9 November 2010

\title{
Screening patients with hypertrophic cardiomyopathy for Fabry disease using a filter-paper test: the FOCUS study
}

\author{
Albert A Hagège, ${ }^{1}$ Eric Caudron, ${ }^{2,3}$ Thibaud Damy, ${ }^{4}$ Raymond Roudaut, ${ }^{5}$ \\ Alain Millaire, ${ }^{6}$ Caroline Etchecopar-Chevreuil, ${ }^{7}$ Thi-Chien Tran, ${ }^{3}$ Firas Jabbour, ${ }^{8}$ \\ Catherine Boucly, ${ }^{8}$ Patrice Prognon, ${ }^{2}$ Philippe Charron, ${ }^{9}$ Dominique P Germain, ${ }^{3,10,11}$ \\ on behalf of the FOCUS study investigators
}

\begin{abstract}
Background Patients with Fabry disease (FD) show left ventricular hypertrophy (LVH) mimicking hypertrophic cardiomyopathy (HCM) of sarcomeric origin and might benefit, if detected early, from specific enzyme replacement therapy. The prevalence of FD in patients with $\mathrm{LVH}$ of $13 \mathrm{~mm}$ or greater, screened using the leucocyte alpha-galactosidase A ( $\alpha$-gal A) activity test, a technique that is difficult to apply routinely, ranged from $0 \%$ to $6 \%$.
\end{abstract}

Objective To screen systematically for FD in patients with a diagnosis of $\mathrm{HCM}$ ( $\mathrm{LVH} \geq 15 \mathrm{~mm}$ ) in primary cardiology practice, a validated, physician-friendly $\boldsymbol{\alpha}$-gal A assay was used on dried blood spots using a filter paper test.

Design and patients $A$ cohort of 392 adults (278 men) followed for HCM were screened for FD. A standard blood test was used for confirmation in nine men in whom the $\alpha$-gal A result was $40 \%$ or less.

Results Four men $(1.5 \% ; 1.8 \%$ of men $\geq 40$ years vs $0 \%$ $<40$ years; all with $\alpha$-gal $A<30 \%$ ), but no women, were diagnosed with FD. Index cases presented with diffuse but asymmetric LVH, with severe obstruction in one case and frequent high-grade atrioventricular conduction block necessitating a pacemaker in three cases. Family screening identified eight additional cases. Genotyping was performed successfully on DNA extracted from the filter papers.

Conclusion In male patients diagnosed as having HCM, pure FD cardiac variants are not exceptional and can be specifically identified using a simple filter-paper test. The sensitivity of this test is low in female patients.

Hypertrophic cardiomyopathies (HCM) are defined by the presence of increased left ventricular thickness or mass in the absence of loading conditions (hypertension, valve disease) sufficient to cause the observed abnormality. ${ }^{1}$ Several hundred mutations involving more than 10 different genes encoding sarcomeric proteins account for approximately $60 \%$ of HCM cases. $^{2}$ While echocardiographic left ventricular hypertrophy (LVH) is the hallmark of the disease, there is a great heterogeneity of symptoms and prognoses among patients with sarcomeric HCM, some having a statistically normal life expectancy and others having a high risk of sudden death, congestive heart failure, or stroke due to atrial fibrillation. ${ }^{2}$
Besides sarcomeric HCM, a complex genetic disorder inherited as a Mendelian autosomal dominant trait, defects in other genes involving general metabolic pathways can also lead to LVH as seen in HCM. Among them, mutations in the $\alpha$-galactosidase A ( $\alpha$-gal A) encoding gene on the $\mathrm{X}$ chromosome may cause Fabry disease (FD), a rare glycosphingolipid storage disorder ${ }^{3}$ suitable for a disease-specific treatment with enzyme replacement therapy (ERT) using recombinant $\alpha$-gal A. ${ }^{4}$ $\mathrm{LVH}$, most often moderate, is present in more than $80 \%$ of men, ${ }^{5}{ }^{6}$ and in one of three to five women ${ }^{3}$ with FD. Its prevalence increases with age ${ }^{67}$ and is usually observed along with other organ failure as renal dysfunction or neurological damage. ${ }^{4} 8$ However, cardiac variants showing exclusive or predominant LVH mimicking sarcomeric HCM have been described in adult patients with FD. ${ }^{3} 6$ When prescribed early enough, ERT can stabilise renal function ${ }^{9}$ and improve $\mathrm{LVH}$ and diastolic function. ${ }^{4} 10$

In previous studies using plasma or leucocyte $\alpha$-gal A activity and/or genotyping to screen systematically for FD cardiac variants in adult patients with apparently unexplained LVH, the prevalence of $\mathrm{FD}$ varies markedly between $0 \%$ and $12 \% .^{7}{ }^{11-14}$ However, both tests are expensive and difficult to implement on a large scale and plasma $\alpha$-gal A activity may lead to both false positive and false negative results. ${ }^{15}$ In addition, these studies often included selected patients from tertiary, specialised centres. Finally, they applied an unusually low echocardiographic threshold (LVH $\geq 13 \mathrm{~mm}$ ) to diagnose HCM, ${ }^{71-14}$ which should be based in sporadic cases on the presence of unexplained LVH of $15 \mathrm{~mm}$ or greater. ${ }^{2} 16$ FOCUS (Fabry $\underline{\text { Or }}$ Cardiomyopathy: Use of a systematic Screening) was a multicentre study that used a simple, physician-friendly filter paper test ${ }^{17}$ for screening FD patients initially diagnosed with HCM in a general cardiology setting in France.

\section{PATIENTS AND METHODS \\ Inclusion criteria}

From July 2006 to July 2008, patients with HCM were recruited in 29 French cardiology centres. Each cardiologist was asked to include up to five patients presenting with HCM of presumed sarcomeric origin, irrespective of the time of diagnosis. After having signed an informed consent document, 
patients were included if they met the following criteria: (1) age 18-79 years; (2) HCM defined as the presence of two-dimensional echocardiographic LVH of unknown cause, such as severe hypertension or valvular aortic stenosis, with a maximum enddiastolic wall thickness of $15 \mathrm{~mm}$ or greater in any segment ${ }^{16}$; and (3) no genetic testing for HCM and no known FD or sarcomeric mutation in the patient and their family. Only patients with mild to moderate hypertension were included in the study as most data suggest that moderate to severe hypertrophy (wall thickness $\geq 15 \mathrm{~mm}$ ) is rare in white people with mild to moderate hypertension. ${ }^{18}$ Left ventricular wall thicknesses were measured directly at end-diastole on echographic two-dimensional parasternal short-axis views (either at the base, papillary muscle or apex levels) using fundamental imaging and taking care to adjust the cut-planes perpendicular to the left ventricular long axis; the greatest thickness as measured at any site within the left ventricular wall using the leading edge method represented the maximum wall thickness. ${ }^{2} 16$

\section{Data collection and diagnostic tests}

Patients' demographic characteristics, HCM-related medical history, echocardiographic measurements and current drug therapy were recorded at inclusion. Blood was drawn from every patient by venipuncture. Five drops from each blood sample were directly spotted on two filter papers. The spots were dried at room temperature for $8-72 \mathrm{~h}$ and then stored at $+2^{\circ}$ to $+6^{\circ} \mathrm{C}$ for up to 2 months before being centrally processed (Laboratoire de génétique médicale, Garches, France); $\boldsymbol{\alpha}$-gal A activity was then measured from dried blood spots (DBS) on filter paper using a fluorimetric method ${ }^{17}$ with modifications as described previously. ${ }^{19}$

When $\alpha$-gal A activity was greater than $40 \%$ compared with the control, a threshold determined from the results obtained from a different genotyped cohort (see below), the test was considered negative and there was no follow-up visit. The relatively high activity threshold was chosen in an attempt to detect FD disease in women, because a high enzymatic activity in women cannot accurately exclude heterozygosity for FD. ${ }^{20}$ When activity was $40 \%$ or less, a second visit was scheduled to answer a questionnaire oriented to characterise signs or symptoms of FD (family history, acute or chronic peripheral neuropathic pain in adolescence, decreased ability to sweat, cornea verticillata, angiokeratoma, gastrointestinal complaints, renal dysfunction, proteinuria, or stroke at age $<55$ years), and confirm FD diagnosis using a leucocyte enzyme activity measure test in men and genotyping in women. To do so, blood samples were shipped to the central laboratory within $96 \mathrm{~h}$. Enzyme activity was assessed by a fluorimetric method on leucocytes, ${ }^{21}$ and genotyping was performed on DNA extracted from the filter papers.

\section{Accuracy of the DBS screening test}

Filter paper test accuracy has been validated previously, being compared with the leucocyte enzyme activity measure test. ${ }^{19} 2122$ Furthermore, we tested the accuracy of the DBS test on an independent cohort of 209 subjects (128 genotyped FD patients 60 men, 68 women - and 81 healthy controls). The enzymatic activity of $\beta$-galactosidase on DBS was found to be normal for all subjects, suggesting the absence of any pre-analytical problem. A positive screening test was defined by an $\alpha$-gal A activity of less than $2.1 \mu \mathrm{mol} / \mathrm{h}$ per litre, which represented $40 \%$ of the median activity of controls $(5.3 \mu \mathrm{mol} / \mathrm{h}$ per litre; mean $\pm \mathrm{SD}$ (range) $5.6 \pm 2$ (2.6-10.7) $\mu \mathrm{mol} / \mathrm{h}$ per litre), allowing us to eliminate false-positive results. In hemizygous men, $\boldsymbol{\alpha}$-gal $\mathrm{A}$ activities were all less than $1.1(0.11 \pm 0.2) \mu \mathrm{mol} / \mathrm{h}$ per litre. In heterozygous women, $\alpha$ gal $\mathrm{A}$ activity was $2.2 \pm 1.7 \mu \mathrm{mol} / \mathrm{h}$ per litre, ranging from 0 to $7.8 \mu \mathrm{mol} / \mathrm{h}$ per litre. Using this threshold ( $\alpha$-gal A $<40 \%$ of controls), the specificity of the test was $100 \%$, whereas the sensitivity was $82 \%$ (100\% in men but only $66 \%$ in women).

\section{PCR conditions}

The PCR were performed as follows: final volume sample $50 \mu \mathrm{l}$; $2 \mathrm{~mm}$ of dried blood from the filter paper as a source of DNA, $25 \mu \mathrm{l}$ water, $2.5 \mathrm{pmol}$ of each primer, and $20 \mu \mathrm{l}$ of Phusion Blood direct PCR (Finnzymes Inc, Massachusetts, USA) were used. An initial denaturation at $95^{\circ} \mathrm{C}$ for 5 min was followed by 35 cycles of $30 \mathrm{~s}$ at $95^{\circ} \mathrm{C}, 30 \mathrm{~s}$ at $58^{\circ} \mathrm{C}, 1 \mathrm{~min}$ at $72^{\circ} \mathrm{C}$, and a final $10 \mathrm{~min}$ at $72^{\circ} \mathrm{C}$.

\section{Genotyping}

Direct sequencing was performed by Beckman Coulter Genomics (Essex, UK). PCR products were purified by Agencourt AMPure XP (Beckman Coulter Genomics). A 3730xl Analyser (Applied Biosystems Inc, California, USA) was used to obtain DNA sequences, which were subsequently handled with Navigator 2.0 software.

\section{Data analysis}

At least 369 patients had to be included in the study to allow an accurate estimation of the prevalence of $\mathrm{FD}$, with an expected value of $4 \%$ and a $95 \%$ CI from $2 \%$ to $6 \%$. When appropriate, results are expressed as mean \pm standard deviation (SD) (range). Groups have been compared using a non-parametric Wilcoxon test for continuous variables and Fisher's exact test for categorical variables.

\section{RESULTS \\ Study population}

Three-hundred and ninety-two patients (278 men) were included in the study (table 1).

A diagnosis of HCM was established at least 5 years earlier in more than $50 \%$ of patients and was new in 124 cases $(31.6 \%)$. Fifty-one patients older than 65 years were hypertensive $(61.5 \%)$ and all of them had been followed for HCM for more than 5 years. A family history of HCM or sudden death was observed in $22 \%$ and $14 \%$ of men, respectively, and in $41 \%$ and $27 \%$ of women. The inheritance pattern a priori excluded the X-linked FD (father-son transmission) in 28 cases. Syncope or presyncope, angina, heart failure and atrial arrhythmias (permanent, sustained, or not) were the most common symptoms. Previous ventricular arrhythmias were not infrequent, with 34 patients having experienced ventricular tachycardia, five having experienced sudden death due to ventricular fibrillation (all implanted with an automatic internal cardiac defibrillator), and four patients with ventricular premature beats of Lown class 3 . Malignant LVH ( $\geq 30 \mathrm{~mm}$ ) was observed in $6 \%$ of men and $3 \%$ of women $(p=0.12)$. During the study, two women died of cardiac causes (one after cardiac transplantation and one of sudden death), three men were resuscitated after sudden death (one with an appropriate shock from his defibrillator), and six patients were hospitalised for worsening heart failure. No endomyocardial biopsy was performed in that cohort.

\section{Screening results}

Among women, all had suitable filter paper tests and enzyme activity greater than $40 \%$ and no FD was diagnosed. Among men, three had non-usable filter paper tests, as a result of 
Table 1 Study group characteristics $(n=392)$

\begin{tabular}{ll}
\hline Variable & \\
\hline Demographics & \\
Age, years, mean \pm SD (range) & $53 \pm 14(18-79)$ \\
$\leq 30$ (women/men), $\mathrm{n}$ & $6 / 19$ \\
$30-40$ (women/men), $\mathrm{n}$ & $9 / 33$ \\
$\geq 40$ (women/men), $\mathrm{n}$ & $99 / 226$ \\
Men, $\mathrm{n}$ (\%) & $278(71)$ \\
Medical history & \\
Time since diagnosis of HCM, years, mean $\pm \mathrm{SD}$ (range) & $8 \pm 9(0-46)$ \\
History of hypertension & $136(34)$ \\
Duration of hypertension, years, mean \pm SD (range) & $12 \pm 10(0-45)$ \\
Family history of HCM & $102(26)$ \\
Family history of sudden death & $71(18)$ \\
Symptoms & \\
NYHA class & \\
I & $118(30)$ \\
II & $187(47)$ \\
III & $82(21)$ \\
IV & $7(2)$ \\
(Pre)syncope & $108(27)$ \\
Angina pectoris & $108(27)$ \\
Atrial arrhythmias & $115(29)$ \\
Ventricular arrhythmias & $43(11)$ \\
No symptoms & $64(16)$ \\
Echocardiography & \\
Maximum left ventricular wall thickness, mm, mean $\pm S D$ & $20 \pm 5(15-40)$ \\
(range) & \\
Left ventricular wall thickness $\geq 30 \mathrm{~mm}$ & $21(5)$ \\
Left ventricular outflow tract gradient $\geq 30 \mathrm{~mm}$ Hg & $121(31)$ \\
\hline Res &
\end{tabular}

Results are shown as number (\%) unless stated otherwise.

HCM, hypertrophic cardiomyopathy; NYHA New York Heart Association.

confirmed conservation problems, and nine had filter paper residual enzyme activities below the defined cut-off of $40 \%-$ markedly decreased $(<17 \%)$ in four cases and slightly below the cut-off value (range 35-39\%) in five cases-leading to a second enzymatic assay performed on leucocytes. Among those nine patients, the five with enzyme activities between $35 \%$ and $39 \%$ on filter papers (i.e. slightly below the initially defined cut-off value for the leucocyte assay) were found to have a leucocyte enzyme activity greater than $40 \%$, while DNA sequencing failed to identify a pathogenic mutation in the GLA gene. In contrast, the remaining four patients with markedly reduced enzyme activity on DBS $(<17 \%)$ were all confirmed as having FD, with a leucocyte enzyme activity between $5 \%$ and $17 \%$. The prevalence of FD was thus $1.5 \%$ (95\% CI 0.4 to 3.8 ) in our cohort of 275 men with interpretable filter paper tests and $1.8 \%$ in men above 40 years of age.

\section{GLA gene mutations}

For patients 1 and 2, sequencing was performed on PCR products obtained after amplification of DNA extracted from leucocyte pellets. For patients 3 and 4, direct sequencing was performed on purified PCR products obtained from direct amplification of DNA eluted from a $3 \mathrm{~mm}$ punch of DBS from filter paper in the PCR mix. Patient 1 was shown to carry a $G$ to $C$ transversion at position c. 486 in exon 3 of the complementary DNA sequence, leading to a missense mutation (p.Trp162Cys). Patients 2 and 3 were both shown to carry a $T$ to $C$ transition at position c. 337 in exon 2 the cDNA sequence of the GLA gene, also leading to a missense mutation (p.Phe113Leu). Interestingly, these two patients - who do not know each other-are both of Portuguese ancestry. Haplotype studies are ongoing to determine whether they are related or not. Patient 4 was shown to carry an A to $G$ transition at position c. 644 in exon 5 of the cDNA sequence (missense mutation p.Asn215Ser). In all cases, sequencing of the rest of the GLA gene revealed no other abnormality.

\section{Characteristics of FD patients}

The mean age of the four men with FD was not significantly different from that of the remaining male patients of the cohort (52 \pm 8 years $(42-59)$ vs $53 \pm 15$ years (18-79), respectively, $\mathrm{p}=0.88$ ). While all patients showed diffuse but asymmetric $\mathrm{LVH}$, a septal to posterior wall ratio greater than 1.5 was not observed. Two patients presented with systolic anterior motion of the mitral valve and outflow tract obstruction, severe in one case, leading to septal ablation (table 2).

Left ventricular ejection fraction was normal in all cases. No FD patients presented with right ventricular hypertrophy or aortic root dilatation; FD patients with systolic anterior motion of the mitral valve showed mild mitral regurgitation, and one FD patient presented with minimal aortic regurgitation. Three patients (1, 2 and 4) underwent permanent pacemaker implantation due to symptomatic high-grade atrioventricular block at 43, 47 and 53 years of age, compared with only one patient in the remaining cohort. No patient reported characteristic signs or symptoms of FD. Moreover, there was no medical history of pain, angiokeratoma, cornea verticillata or proteinuria. On a systematic brain magnetic resonance imaging scan, one patient had a small lacunar infarction, whereas all the others tested normal. Another patient showed mild proteinuria, with an estimated glomerular filtration rate normal for his age. Pedigree analysis identified two half-brothers at risk of FD for patient number 4, and sequencing of the GLA gene confirmed the diagnosis of FD through the identification of a missense mutation in exon 5 of the gene (p. N215S). Interestingly, diffuse but asymmetric LVH of unknown cause existed for both of them. Furthermore, six heterozygous women were identified through pedigree analysis in three families.

\section{DISCUSSION}

In $\mathrm{FD}, \alpha$-gal A deficiency causes progressive accumulation of globotriaosylceramide and related glycosphingolipids in heart

Table 2 Clinical and genetic characteristics of men diagnosed with Fabry disease

\begin{tabular}{|c|c|c|c|c|c|c|c|c|c|c|c|}
\hline $\begin{array}{l}\text { Patient } \\
\text { number }\end{array}$ & $\begin{array}{l}\text { Age } \\
\text { (years) }\end{array}$ & $\begin{array}{l}\text { (Pre) } \\
\text { syncope }\end{array}$ & Angina & NYHA class & FH & HT & PM & AF & VT & $\begin{array}{l}\text { Maximum WT } \\
\text { (mm) (IVS/PW) }\end{array}$ & $\begin{array}{l}\text { SAM/gradient } \\
\text { (mm } \mathrm{Hg})\end{array}$ \\
\hline 1 & 59 & + & 0 & 1 & 0 & 0 & Yes & 0 & 0 & $21(1.4)$ & no/0 \\
\hline 2 & 48 & 0 & + & III & 0 & + & No & + & + & $19(1.3)$ & yes/30 $\left(140^{*}\right)$ \\
\hline 3 & 59 & + & 0 & ॥ & 0 & 0 & Yes & 0 & 0 & $20(1.3)$ & no/0 \\
\hline 4 & 41 & + & + & I & 0 & 0 & Yes & 0 & 0 & $21(1.4)$ & yes/30 \\
\hline
\end{tabular}

* Maximal gradient at exercise before septal embolisation. None of the patients had any other features of Fabry disease outside the heart.

$\mathrm{AF}$, atrial fibrillation or arrhythmias; $\mathrm{FH}$, known family history of hypertrophic cardiomyopathy or Fabry disease; gradient, peak outflow tract gradient; $\mathrm{HT}$, hypertension; IVS/PW, ratio of septal thickness on posterior wall thickness; NYHA, New York Heart Association; PM, permanent pacemaker for high-degree atrioventricular block; SAM, systolic anterior motion of the mitral valve; VT, ventricular tachycardia; WT, end-diastolic left ventricular wall thickness. 
tissues, such as cardiomyocytes, conduction system cells, valvular fibrocytes and both vascular endothelial and smooth muscle cells. This heart tissue accumulation potentially leads to irreversible cardiac damage. Cardiac FD manifestations include LVH, arrhythmias, conduction defects and, more rarely, coronary artery disease (mainly due to small-vessel disease). ${ }^{3}$ It contributes to a reduced life expectancy of affected patients due to malignant arrhythmias and heart failure. Whereas, in parallel with multivisceral involvement, nearly all FD patients will develop LVH, the extent of which both increases with age and is accompanied by a progressive reduction in left ventricular function. LVH may also be the predominant feature of the disease in some patients presenting as cardiac variants, even if the existence of pure cardiac variants remains controversial. ${ }^{23}$ Previous systematic screening of FD has been performed using $\alpha$ gal A enzyme activity measured in leucocytes with the fluorogenic substrate 4-methylumbelliferyl- $\alpha$-D-glucopyranoside, currently the gold standard for diagnosing FD in male patients. ${ }^{7} 1114$ More recently, $\boldsymbol{\alpha}$-gal A enzyme activity dosage on DBS using a filterpaper test has been proposed as an alternative diagnostic test, ${ }^{17}$ and has been found to be as accurate as assays using leucocyte samples. ${ }^{19} 22$ The accuracy of this test is also demonstrated by results currently reported in a separate genotyped cohort (see Patients and methods section), with both sensitivity and specificity reaching $100 \%$ in men, whereas in women, as expected, sensitivity was lower (66\%). However, the diagnosis of FD in women can occasionally be made using this method in the cases in which a markedly decreased residual enzyme activity is evidenced, and thus women were included in the current study. ${ }^{24}$ Moreover, those samples are easy to transport and are stable at room temperature for at least 20 days, making them suitable for screening patients at risk of FD. Leucocyte $\boldsymbol{\alpha}$-gal A enzyme activity assays can be used to confirm positive filter paper test results and verify negative results in patients with a clinical suspicion of FD.

The novel aspect of this study is the use of blood spot analysis from physician-friendly filter papers as a practical way of conducting the screening. This was the source of the enzyme assay and could also be used as a source of DNA. Moreover, the prevalence of FD in individuals diagnosed with HCM was based on the consensual LVH diagnosis threshold $(15 \mathrm{~mm})$ in a large number of primary cardiology centres. The prevalence of FD is more frequent in male patients with late onset ( $\geq 40$ years) $\mathrm{HCM}^{7}$; accordingly, in the current study, no men less than 40 years of age were diagnosed with FD, compared with $1.8 \%$ of those aged over 40 years. It also demonstrates the accuracy of screening with the filter paper test, confirmed by both the gold standard enzymatic assay on white blood cells and genotyping (specificity $100 \%$ if a $30 \%$ threshold for residual $\alpha$-gal A activity is used). The filter paper test failed to detect any female patient with FD, but heterozygotes may have been missed as no systematic sequencing was carried out and as the test has a low sensitivity in women. From a practical point of view, the risk that a woman with clinically significant FD may not be diagnosed by a screening test based primarily on $\alpha$-gal A enzyme activity measurement remains high, ${ }^{14}$ and justifies efforts to collect clinical signs or medical history that should encourage first-line genotyping. In a recent cohort using systematic genetic screening in $90 \mathrm{HCM}$ probands, 59 without sarcomere gene mutations, $\alpha$-gal A mutations were found in three of $90(3 \%)$ HCM families and in two of $20(10 \%)$ women without sarcomere gene mutations; none of the probands presented other indices of FD. ${ }^{25}$ This finding supports systematic testing for FD and underlines the fact that enzyme measurements are sufficient in men, but genetic testing is needed in women. Finally, genotyping was performed for the first time on PCR products obtained from DNA eluted directly from filter paper (figure 1). This may prove useful in the future for all cases for which obtaining a second sample may be difficult as well as for the genotyping of women for whom the enzymatic assay is not satisfactory and no efficient screening alternative is currently available.

Our results are in line with data from Montserrat et $a l^{13}$ in which a prevalence of FD of $1 \%$ has been estimated in a population of 508 men and women with HCM of presumed sarcomeric origin. Others cardiac variants have been identified in cohorts of patients with unexplained LVH of $13 \mathrm{~mm}$ or greater and systematically screened for $\mathrm{FD}$, with an overall prevalence of approximately $3 \%$ in men (up to $6 \%$ in those aged above 40 years) $)^{71}$ and up to $12 \%$ in women (in a single study for which methodological bias cannot be excluded).${ }^{14}$ Conversely, no patients with FD were found in highly selected groups with HCM, either in severely symptomatic obstructive patients who underwent surgical procedures to relieve the gradient, ${ }^{26}$ or in

Figure 1 Direct sequencing of polymerase chain reaction products obtained from amplification of DNA eluted directly from the $3 \mathrm{~mm}$ punch of dried blood spot from filter paper (patient 3). Patient 3 was shown to carry a $T$ to $C$ transition at position c. 337 in the cDNA sequence (arrow). This nucleotide substitution alters the codon (TTT) for phenylalanine to the codon (CTT) for leucine at position 113 of the $\alpha$-galactosidase A protein (p. Phe113Leu). Despite scanning of the rest of the gene, no other sequence abnormality was found. 
systematically genotyped patients. ${ }^{12}$ The apparent discrepancies between these different results may be related to the different methods used (type of population selected, assay methods).

While echographic asymmetric LVH has been described in patients with FD, diffuse, concentric and homogeneous LVH is the more frequently reported pattern. ${ }^{3} 6$ The current study demonstrates, quite unexpectedly when compared with a previous study, ${ }^{24}$ that cardiac variants may show asymmetric LVH and may go unrecognised for decades. However, a septal to posterior wall ratio greater than 1.5 was not observed. Severe obstruction leading to septal ablation is infrequent, but has been reported. ${ }^{27}$ Syncope associated with high-grade atrioventricular block seems to be a rather specific characteristic of the FD cardiac variant in this study, as three out of four patients diagnosed with FD presented with a syncopal high-grade atrioventricular blockage leading to permanent pacemaker implantation.

\section{Conclusions}

In men diagnosed with HCM, cardiac variants of FD are not exceptional and might benefit from specific ERT. These patients may be screened systematically for FD with a simple fluorimetric method using DBS on filter paper test, which has become a very efficient first-line diagnostic test for screening in a general cardiology setting. High-degree atrioventricular block is frequent in that population. As the outcome of specific ERT is less efficient in advanced cases of FD, before extensive myocardial fibrosis is present, ${ }^{428}$ such a systematic screening using a simple test appears to be clinically relevant.

\footnotetext{
Acknowledgements The authors acknowledge the Centre d'Investigations Cliniques (Michel Azizi, MD, Anne Blanchard, MD), Hôpital Européen Georges Pompidou, Paris. The authors would like to thank Olivier Charansonney for his assistance in collecting and interpreting the data, and they thank the centres (listed in alphabetical order) and investigators participating in the FOCUS study, and the 'Groupe Cardiomyopathies et Insuffisance Cardiaque' of the French Society of Cardiology: Besançon-Centre Hospitalier Universitaire Saint-Jacques (Yvette Bernard); Hôpital Jean Minjoz (Joanna Oettinger, Marie-France Seronde); BoulogneBillancourt-Hôpital Ambroise-Paré (Florence de Roquefeuil, Olivier Dubourg, Laurent Gouya, Laure Revault D'allones); Brest-Hôpital de la Cavale Blanche (Yannick Jobic); Cergy-Pontoise-Centre Hospitalier René Dubos (Patrick Jourdain); Clamart-Hôpital Militaire Percy (Eric Perrier); Clermont-Ferrand-Centre Hospitalier Universitaire G Montpied (Aimé Théodore Amonchot, Bernard Citron); Créteil-Hôpital Henri Mondor (Thibault Damy, Jean-Luc Dubois-Rande, Philippe Lecorvoisier); Dijon - Hôpital du Bocage (Jean-Christophe Eicher, Pierre Henry Gacon, Isabelle L'Huillier); La Martinique-Centre Hospitalier La Meynard (Elisabeth Sarrazin); La Réunion-Centre Hospitalier Sud Réunion (Christophe Jeannot, Catherine Mimran); Lille-Hôpital Cardiologique (Alain Millaire); Limoges-Centre Hospitalier Universitaire Régional Dupuytren (Caroline Etchecopar-Chevreuil, Jérôme Lesage); Lyon-Hôpital Cardiologique Louis Pradel (Patrice Bouvagnet, Geneviève Derumeaux); Montpellier-Hôpital Arnaud de Villeneuve (Dinh Thien-Tri Cung, JeanMarc Davy, Catherine Sportouch-Dukhan); Nantes-Centre Hospitalier Universitaire $G$ et $R$ Laënnec (Jean-Pierre Gueffet, Jean-Marc Langlard, To Ngoc Tram To, JeanNoël Trochu); Nîmes-Centre Hospitalier Universitaire Caremeau (Stéphane Cade); Paris - Hôpital Bichat (Marie-Claude Aumont, Guillaume Jondeau); Hôpital Européen Georges Pompidou (Michel Desnos, Albert A Hagege); Hôpital Lariboisière (Christophe Chavelas, Damien Logeart); Hôpital de La Pitié Salpêtrière (Philippe Charron, Richard Isnard, Françoise Pousset); Hôpital Militaire du Val de Grâce (Christian Bergez); Pessac-Hôpital Cardiologique du Haut Lévêque (Patricia Reant, Raymond Roudaut); Rennes - Centre Hospitalier Universitaire Pontchaillou (Erwan Donal, Renaud Gervais, Christophe Leclerq); Rouen-Hôpital Charles Nicolle (Fabrice Bauer, Mathieu Lemercier); Strasbourg - Centre Hospitalier Universitaire Hautepierre (Gérald Roul); Suresnes-Hôpital Foch (Alain Guiomard, Franck Haziza, Stéphanie Russel); Toulouse-Hôpital Rangueil (Michel Galinier, Michel Jean, Pierre Massabuau, Jérôme Roncalli); Vandoeuvre Les Nancy-Centre Hospitalier Universitaire de Brabois (Yves Julliere). The following individuals provided expert technical help in the laboratory: Pierre Chaminade, Isabelle Delhom, Ramzi Ezarragui, Coralie Favier, Cindy Gallerne, Laetitia Lacastaigneratte, Maryline Le Boulbin, AnneIsabelle Martin-Mista, Gwen Page and Jean-François Perrault. This study was promoted by the French Society of Cardiology, with particular help from the Commission Promotion et Recherche Clinique (Dr Anissa Bouzamondo and Emilie Nonnotte).
}

Funding This study was supported by Genzyme SAS, Saint-Germain en Laye, France and the Centre de Référence de la maladie de Fabry et des maladies héréditaires du tissu conjonctif, CHU Raymond Poincaré, Garches, France.

Competing interests AAH and DPG have received research and consultancy funding from Genzyme SAS, Saint-Germain en Laye, France.

\section{Patient consent Obtained.}

Ethics approval This study was conducted with the approval of the ethical committee of Pitié Salpêtrière, Paris, no 11-06, favourable advice 20 March 2006, France.

Provenance and peer review Not commissioned; externally peer reviewed.

\section{REFERENCES}

1. Elliott $\mathbf{P}$, Andersson B, Arbustini E, et al. Classification of the cardiomyopathies: a position statement from the European Society of Cardiology Working Group on Myocardial and Pericardial Diseases. Eur Heart J 2008;29:270-6.

2. Maron BJ, McKenna WJ, Danielson GK, et al. American College of Cardiology/ European Society of Cardiology Clinical Expert Consensus Document on Hypertrophic Cardiomyopathy. A report of the American College of Cardiology Foundation Task Force on Clinical Expert Consensus Documents and the European Society of Cardiology Committee for Practice Guidelines. Eur Heart J 2003;24:1965-91.

3. Linhart A, Kampmann C, Zamorano JL, et al. Cardiac manifestations of Anderson-Fabry disease: results from the international Fabry outcome survey. Eur Heart J 2007;28:1228-35.

4. Weidemann $\mathbf{F}$, Niemann $M$, Breunig $F$, et al. Long-term effects of enzyme replacement therapy on Fabry cardiomyopathy: evidence for a better outcome with early treatment. Circulation 2009;119:524-9.

5. MacDermot KD, Holmes A, Miners AH. Anderson-Fabry disease: clinical manifestations and impact of disease in a cohort of 98 hemizygous males. J Med Genet 2001;38:750-60.

6. Kampmann C, Linhart A, Baehner F, et al. Onset and progression of the Anderson-Fabry disease related cardiomyopathy. Int J Cardiol 2008;130:367-73.

7. Sachdev B, Takenaka T, Teraguchi H, et al. Prevalence of Anderson-Fabry disease in male patients with late onset hypertrophic cardiomyopathy. Circulation 2002; 105:1407-11

8. Waldek S, Patel MR, Banikazemi M, et al. Life expectancy and cause of death in males and females with Fabry disease: findings from the Fabry Registry. Genet Med 2009;11:790-6.

9. Germain DP, Waldek S, Banikazemi M, et al. Sustained, long-term renal stabilization after 54 months of agalsidase beta therapy in patients with Fabry disease. J Am Soc Nephrol 2007;18:1547-57.

10. Kovacevic-Preradovic T, Zuber M, Attenhofer Jost $\mathrm{CH}$, et al. Anderson-Fabry disease: long-term echocardiographic follow-up under enzyme replacement therapy. Eur J Echocardiogr 2008;9:729-35

11. Nakao S, Takenaka T, Maeda M, et al. An atypical variant of Fabry's disease in men with left ventricular hypertrophy. N Engl J Med 1995;333:288-93.

12. Arad M, Maron BJ, Gorham JM, et al. Glycogen storage diseases presenting as hypertrophic cardiomyopathy. N Engl J Med 2005;352:362-72.

13. Monserrat L, Gimeno-Blanes JR, Marin F, et al. Prevalence of Fabry disease in a cohort of 508 unrelated patients with hypertrophic cardiomyopathy. J Am Coll Cardiol 2007; 50:2399-403.

14. Chimenti C, Pieroni M, Morgante E, et al. Prevalence of Fabry disease in female patients with late-onset hypertrophic cardiomyopathy. Circulation 2004;110:1047-53

15. Andrade J, Waters PJ, Singh RS, et al. Screening for Fabry disease in patients with chronic kidney disease: limitations of plasma alpha-galactosidase assay as a screening test. Clin J Am Soc Nephrol 2008;3:139-45.

16. Maron MS, Maron BJ, Harrigan C, et al. Hypertrophic cardiomyopathy phenotype revisited after 50 years with cardiovascular magnetic resonance. J Am Coll Cardiol 2009;54:220-8.

17. Chamoles NA, Blanco M, Gaggioli D. Fabry disease: enzymatic diagnosis in dried blood spots on filter paper. Clin Chim Acta 2001;308:195-6.

18. Shapiro LM, Kleinebenne A, McKenna WJ. The distribution of left ventricular hypertrophy in hypertrophic cardiomyopathy: comparison to athletes and hypertensives. Eur Heart J 1985;6:967-74.

19. Caudron E, Moliere D, Zhou JY, et al. [Recent advances of Fabry disease screening for at risk population]. Med Sci (Paris) 2005:21:48-50.

20. Mayes JS, Scheerer JB, Sifers RN, et al. Differential assay for lysosomal alphagalactosidases in human tissues and its application to Fabry's disease. Clin Chim Acta 1981;112:247-51.

21. Lukacs Z, Keil A, Kohlschutter A, et al. The ratio of alpha-galactosidase to betaglucuronidase activities in dried blood for the identification of female Fabry disease patients. J Inherit Metab Dis 2005;28:803-5.

22. Lukacs $\mathbf{Z}$, Hartung $\mathrm{R}$, Beck $\mathrm{M}$, et al. Direct comparison of enzyme measurements from dried blood and leukocytes from male and female Fabry disease patients. $J$ Inherit Metab Dis 2007;30:614.

23. Germain DP. A new phenotype of Fabry disease with intermediate severity between the classical form and the cardiac variant. Contrib Nephrol 2001:234-40. 
24. Linthorst GE, Bouwman MG, Wijburg FA, et al. Screening for Fabry disease in high risk populations: a systematic review. J Med Genet 2010;47:217-22.

25. Havndrup 0, Christiansen M, Stoevring B, et al. Fabry disease mimicking hypertrophic cardiomyopathy: genetic screening needed for establishing the diagnosis in women. Eur J Heart Fail 2010;12:535-40.

26. Ommen SR, Nishimura RA, Edwards WD. Fabry disease: a mimic for obstructive hypertrophic cardiomyopathy? Heart 2003;89:929-30.
27. Magage S, Linhart A, Bultas J, et al. Fabry disease: percutaneous transluminal septal myocardial ablation markedly improved symptomatic left ventricular hypertrophy and outflow tract obstruction in a classically affected male. Echocardiography 2005:22:333-9.

28. Mehta A, Beck M, Elliott P, et al. Enzyme replacement therapy with agalsidase alfa in patients with Fabry's disease: an analysis of registry data. Lancet 2009;374 1986-96. 